\title{
Hydrogen Rearrangement to and from Radical z Fragments in Electron Capture Dissociation of Peptides
}

\author{
Mikhail M. Savitski, ${ }^{*}$ Frank Kjeldsen,, Michael L. Nielsen, \\ and Roman A. Zubarev \\ Laboratory for Biological and Medical Mass Spectrometry, Uppsala University, Uppsala, Sweden
}

\begin{abstract}
Hydrogen rearrangement is an important process in radical chemistry. A high degree of $\mathrm{H}$. rearrangement to and from $z$. ionic fragments (combined occurrence frequency $47 \%$ compared with that of $z \cdot$ ) is confirmed in analysis of 15,000 tandem mass spectra of tryptic peptides obtained with electron capture dissociation (ECD), including previously unreported double $\mathrm{H}$. losses. Consistent with the radical character of $\mathrm{H} \cdot$ abstraction, the residue determining the formation rate of $z^{\prime}=z \cdot+\mathrm{H} \cdot$ species is found to be the $\mathrm{N}$-terminal residue in $z \cdot$ species. The size of the complementary $c_{m}{ }^{\prime}$ fragment turned out to be another important factor, with $\mathrm{z}^{\prime}$ species dominating over $z$. ions for $m \leq 6$. The $\mathrm{H}$. atom was found to be abstracted from the side chains as well as from $\alpha$-carbon groups of residues composing the $c^{\prime}$ species, with Gln and His in the $c^{\prime}$ fragment promoting $\mathrm{H}$. donation and Asp and Ala opposing it. $\mathrm{Ab}$ initio calculations of formation energies of $\cdot A$ radicals ( $A$ is an amino acid) confirmed that the main driving force for $\mathrm{H}$ - abstraction by $z$. is the process exothermicity. No valid correlation was found between the $\mathrm{N}-\mathrm{C}_{\alpha}$ bond strength and the frequency of this bond cleavage, indicating that other factors than thermochemistry are responsible for directing the site of ECD cleavage. Understanding hydrogen attachment to and loss from ECD fragments should facilitate automatic interpretation ECD mass spectra in protein identification and characterization, including de novo sequencing. (J Am Soc Mass Spectrom 2007, 18, 113-120) @ 2007 American Society for Mass Spectrometry
\end{abstract}

$\mathrm{I}$ n proteomics analysis, tandem mass spectrometry (MS/MS) is the primary method of identification of proteins expressed in cells, tissue, and organisms [1-3]. In the bottom-up approach, isolated and prefractionated proteins are digested with trypsin, and the tryptic peptide mixtures are subjected to separation with liquid chromatography (LC) followed by on-line electrospray ionization (ESI) and MS/MS analysis. It has been shown recently that MS/MS using electron capture dissociation (ECD [4] as a complement to conventional collision-activated dissociation (CAD) greatly increases the specificity of the database search [5] and improves de novo sequencing [6].

Dications are the most important ionic species when tryptic peptides are analyzed by ESI, as most peptide ions appear in preferentially $2+$ charge state [5]. Understanding the fragmentation behavior of these species is important for extracting maximum sequence information from MS/MS spectra [7]. We analyzed the database of 15,000 high-resolution ECD

Published online October 23, 2006

Address reprint requests to Dr. Mikhail M Savitski, Laboratory for Biological and Medical Mass Spectrometry, Uppsala University, Box 583, Uppsala S-751 23, Sweden. E-mail: Mikhail.Savitski@bmms.uu.se

* These two authors contributed equally to this article. mass spectra of tryptic peptide dications to study details of the processes concomitant to the primary $\mathrm{N}-\mathrm{C}_{\alpha}$ bond cleavage in ECD that leads to "classical" $c^{\prime}$ and $z$. fragment ions. Formation of other, less usual fragments introduces uncertainty in determination of fragment masses that complicates peptide sequencing. Understanding the mechanism of hydrogen atom transfer in ECD is important for reducing this uncertainty. Recently, O'Connor et al. have reported a detailed study performed on two peptides [8]. They found extensive hydrogen rearrangement and H/D scrambling and concluded that these effects are best explained by the presence of long-lived intermediate radical complexes. In terms of specific behavior of individual amino acids, they found glycine $\alpha$-carbons to readily lose hydrogens, while aspartic acid showed an opposite trend. Here, we performed extensive analysis of occurrence frequencies of hydrogen gain and losses from $z$. fragment ions. These ions constituted a majority of ECD fragments, consistent with the neutralized charge at the less basic N-terminal site $[9,10]$. The most abundant fragments $z_{k-m}$, where $k$ is the peptide size are observed for $m=4$ [11]. Thus the data were collected for $3 \leq m \leq 5$, if not indicated otherwise. 

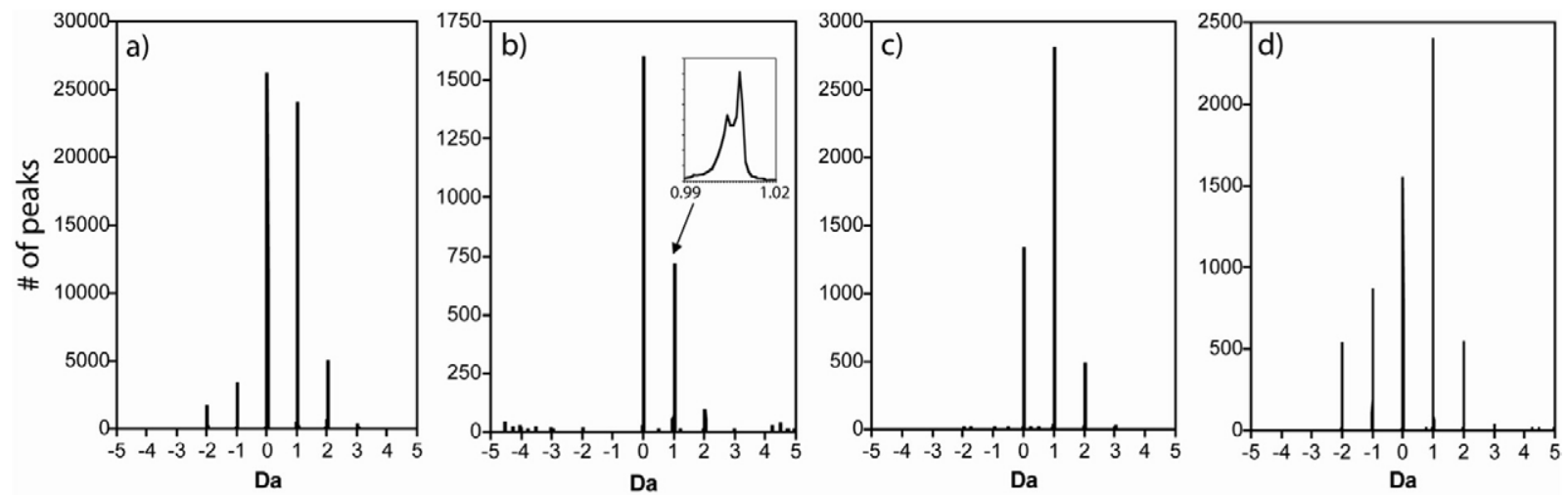

Figure 1. The histogram of the frequency of occurrence of peaks in the $z$-ion cluster, $\Delta \mathrm{M}=0$ corresponds to $z$. fragment, positive $\Delta \mathrm{M}$ values to mass addition, negative values to mass abstraction: (a) total histogram; (b)-(d) histogram for the following amino acids being C-terminal to the cleavage site: (b) Ile; (c) Gly; (d) Ser. Inset in (b) is a zoom-in of the +1 Da region showing separation of the $z^{\prime}$ and ${ }^{13} \mathrm{C}$ contributions.

\section{Experimental}

\section{Mass Spectrometry}

The data were collected from the proteomics analysis of lysates of human cell lines and E. coli (all samples are from Sigma Aldrich, St. Louis, MO) performed on an LTQ FT, 7 tesla mass spectrometer (Thermo Electron, Bremen, Germany) using consecutive ECD and CAD fragmentation of peptides eluting from the analytical column of a nano-LC system (Agilent 1100, Waldbrann, Germany). The peptides were identified by Mascot search engine (Matrix Sciences, London, UK). The details of the experimental procedure and data processing are described in references $[5,11]$.

\section{Ab Initio Calculations}

Standard density functional theory calculations were performed with Gaussian 03 suite of programs. Optimized geometries of the model species relevant to this study were obtained using the B3LYP level of theory and 6-31 + G(d) basis set. For higher accuracy, singlepoint energies of optimized structures were calculated with the same level of theory using 6-311 $+\mathrm{G}(2 \mathrm{~d}, 2 \mathrm{p})$ basis set after adding both polarization and diffuse functions for heavy atoms and hydrogens. The use of diffuse functions is required for structure optimization of electron-rich radical molecules $[12,13]$. The harmonic vibrational frequencies of stationary points on the potential energy surface (PES) were calculated at the same level of theory as used for their optimization to identify local minima (all real frequencies). Zero-point energies (ZPE) were computed by adjusting harmonic frequencies by an appropriate correction factor of 0.9804 for the B3LYP level of theory. The zero-point corrected energies were then combined with the total electronic energies resulting from the geometry optimizations.

\section{Results}

Before $\mathrm{H} \cdot$ transfer analysis, each peptide sequence was reliably identified by a database search [5] and the fragment identity was assigned using high mass accuracy ( \pm 1 to $2 \mathrm{mDa}$ ) of Fourier transform mass spectrometry (FTMS). Figure 1a shows the overall frequency of occurrence of peaks in the $z$-ion cluster.

The most frequent peak at $0 \mathrm{Da}$ is due to $z$. ion resulting from the primary $\mathrm{N}-\mathrm{C}_{\alpha}$ bond cleavage in the aminoketyl radical formed upon electron capture [4]:

$$
\begin{aligned}
& \mathrm{R}_{\mathrm{n}+1^{-}}(\mathrm{COH})-\mathrm{NH}-\mathrm{CH}\left(\mathrm{R}_{\mathrm{n}} \mathrm{H}^{+}\right)^{\prime} \rightarrow \underset{c_{m^{\prime}}}{\mathrm{R}_{\mathrm{n}+1^{-}}(\mathrm{COH})} \\
& =\mathrm{NH}+\underset{z_{\mathrm{n}^{+}} .}{\mathrm{CH}}\left(\mathrm{R}_{\mathrm{n}} \mathrm{H}^{+}\right)^{\prime}
\end{aligned}
$$

Note that the index numeration in eq 1 and the rest of the paper (if not indicated otherwise) is from the C-terminus, as the ionic species under consideration are the C-terminal $z$ ions. The theoretical mass of the $z$ ion was used as the origin of the horizontal axis in Figure 1. The peak $1 \mathrm{Da}$ heavier than $z \cdot$ is a doublet (Figure $1 \mathrm{~b}$ ) consisting of a ${ }^{13} \mathrm{C}$ contribution $(+1.003 \mathrm{Da})$ and a $\mathrm{H}$. addition to $z \cdot(+1.008 \mathrm{Da})$. Due to high mass accuracy of the FTMS instrument used, the doublet on the histogram is sufficiently resolved for the occurrence frequency of $z^{\prime}=\mathrm{Lz} \cdot+\mathrm{H}$. ions to be measured. The next peak $(+2 \mathrm{Da})$ is primarily due to isotopes of $\mathrm{C}, \mathrm{O}$, and $\mathrm{S}$. This peak bears no sign of double $\mathrm{H}$ - addition. The peaks left to $z$ are due to $\mathrm{H}$ - abstraction, which is witnessed by their mass shifts, -1.008 and $-2.016 \mathrm{Da}$. Double $\mathrm{H} \cdot$ abstraction from amino acids other than proline [14] has not been reported in ECD literature. Overall, the frequency of occurrence of $\mathrm{H}$. addition or loss is $47 \%$ compared with that of the $z$. ion which makes $\mathrm{H}$ - rearrangement to/from the radical $z$. fragment (reported earlier in ECD literature [15-17] the most important secondary process in ECD. Here, we 


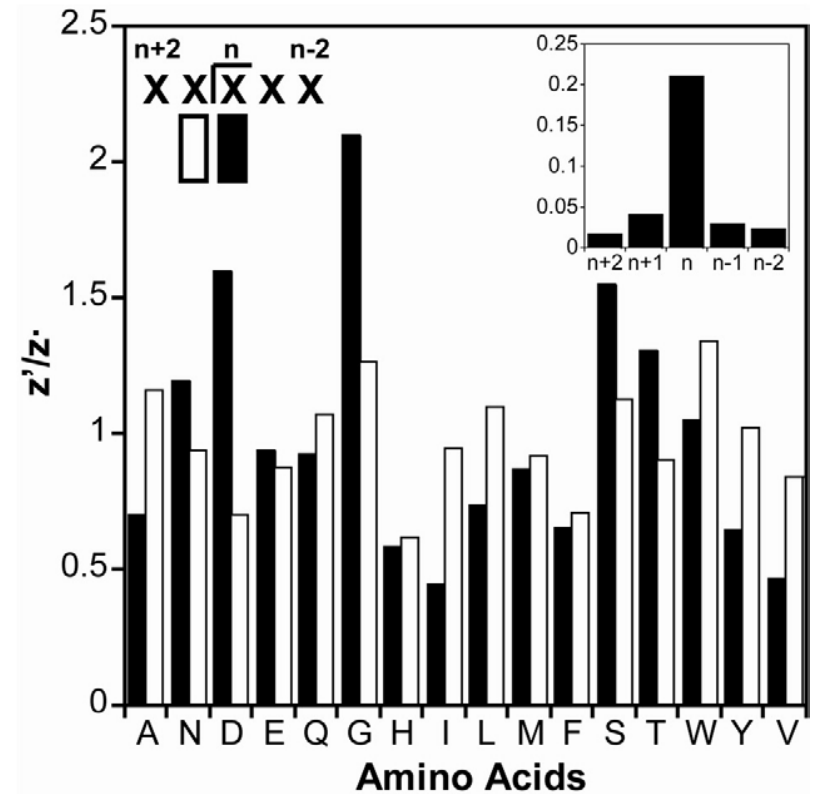

Figure 2. Ratios of the frequencies of occurrence of $z^{\prime}$ and $z$. fragments for residues $\mathrm{C}$-terminal (filled columns) and $\mathrm{N}$-terminal (open columns) to the cleavage site. Inset: variance of the $z^{\prime} / z$. values for residues in different positions: $n$ corresponds to position adjacent to the cleavage from the C-terminal side. Numeration is from the C-terminus to reflect the length of C-terminal $z$ ions.

studied this process from the point of view of the amino acid context. There is a strong dependence of this process upon the amino acid residue adjacent to the cleavage site: fragments with Ile show much more modest $\mathrm{H}$ - addition (Figure 1b) compared with Gly (Figure 1c). While neither one of these two residues shows signs of $\mathrm{H}$. abstraction, the terminal Ser in $z$. leads to abundant abstraction of one and two hydrogen atoms (Figure 1d).

\section{$H \cdot$ Addition to $z$}

Figure 2 presents the ratios of frequencies of occurrences of $z^{\prime}$ and $z$. ions for 16 amino acids adjacent to the $\mathrm{N}-\mathrm{C}_{\alpha}$ cleavage site, excluding the rare Lys, Arg, Cys, and Pro in these positions.

The importance of the amino acid identity in a specific position relative to the cleavage site can be assessed by calculating the variance of the $z^{\prime} / z$. ratios for the 16 amino acids [11]. If the identity of an amino acid in that position affects the $z^{\prime} / z \cdot$ ratio, the ratios for some amino acids are expected to deviate significantly from their own average value, thus showing a large variance. If, on the contrary, the identity of the amino acid is not important, all $z^{\prime} / z$. values should be more or less similar and the variance will be small. Analysis of the variance values shown in the inset in Figure 2 reveals that by far the most important residue for $\mathrm{H} \cdot$ attachment is the one adjacent to the cleavage from the C-terminal side (this position is denoted as $n$ ). The variance at this position is five times greater than that of the next-important resi- due, $n+1$, adjacent to the cleavage from the $\mathrm{N}$-terminal side. On the individual residue level, Gly, Asp, Ser, and Thr in the C-terminal position to the cleavage site as well as Trp and Gly in the N-terminal position promote $\mathrm{H}$ - acceptance by a $z \cdot$ ion. At the same time, Ile, Val, and His in the C-terminal position as well as His, Asp, and Phe in the N-terminal positions discourage it.

In general, we found variance analysis very useful in determining the relative importance of amino acids in different positions for fragmentation processes. In reference [11], variance analysis was used to confirm that in collisional activation the position $n+1$ is the most important one for $\mathrm{C}-\mathrm{N}$ cleavage. But vigorous statistics for the variance analysis is not available. For instance, there is no rational significance threshold for the variance values. Thus, small values for $n-2$ and $n+2$ determine the background level. Against this background, the value for $n+1$ shows a significant increase, while a slight increase for $n-1$ may be a fluctuation. On the other hand, the value for $n-2$ is also elevated compared to $n+2$ and, thus, the propagation of a radical towards the $\mathrm{C}$-terminus in $z$. ions, as in the radical cascade mechanism by $\mathrm{O}^{\prime}$ Connor and coworkers [16], cannot be excluded.

\section{$H \cdot$ Abstraction from $z$}

The hydrogen loss from $z$ ions was found to be very abundant when Thr was adjacent to the radical site (position $n$ ), and prominent when Ser was present in the same position (Figure 3, left panel).

Some $\mathrm{H}$ - loss occurs also for Trp, Phe, and Tyr in the $n$-th position. The identity of the amino acid N-terminal to the cleavage site (position $n+1$ ) was much less important (Figure 3, right panel).

\section{Discussion}

\section{$N-\mathrm{C}_{\beta}$ Cleavage Direction}

The above results can be interpreted based on the known ECD processes. The variance analysis (Figure 2, inset) showing that the identity of the amino acid in the


Figure 3. Ratios of $\mathrm{H} \cdot$ loss occurrence frequencies (filled columns: single loss, open columns: double $\mathrm{H} \cdot$ loss) and occurrence frequencies of $z$ ions (including both $z$ and $z^{\prime}$ ) for residues C-terminal (left) and N-terminal (right) to the cleavage site. 


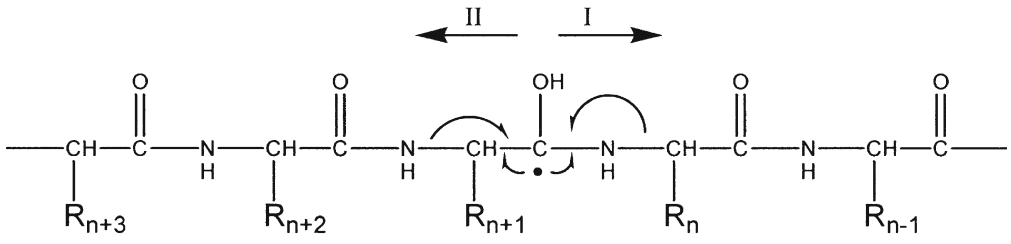

Scheme 1

position $n-1$ (second N-terminal in the $z$. ion) is of relatively low importance is an important result in understanding whether $z^{\prime}$ species are formed as a result of $\mathrm{H}$. addition after the cleavage, as conventionally assumed (and supported by data of $\mathrm{O}^{\prime}$ Connor et al. [8], or by direct $\mathrm{N}-\mathrm{C}_{\alpha}$ cleavage, as has been suggested elsewhere in the literature [16]. In the first case, "classical" right-side cleavage according to reaction eq 1 and pathway I in Scheme 1 result in $z_{n}$. fragments. In the latter case, the process of $\mathrm{N}-\mathrm{C}_{\alpha}$ bond cleavage proceeds left of the aminoketyl radical at the $n$-th residue (pathway II), leading to the formation of $z_{\mathrm{n}+1}$ 'fragments:

It follows from Scheme $\mathbf{1}$ that if the pathways (I) and (II) compete with each other, then the rate of $z_{n^{\prime}}$ formation should be determined by both the residues $n$ and $n+1$. However, the variance analysis (Figure 2, inset) shows much smaller influence of the residue in the position $n+1$ compared with $n$. This result supports the pathway I and formation of $z^{\prime}$ ions by hydrogen atom transfer from the $c^{\prime}$ species inside a shortlived (relative to the observation scale) complex of complementary fragments [8, 18-20]:

$$
\left[c^{\prime}+z^{\prime}\right] \text { complex } \rightarrow\left[c+z^{\prime}\right] \text { complex } \rightarrow c^{\prime}+z^{\prime}
$$

\section{Lifetime of the Complex}

As experimental evidence for the validity of eq 2, the ratio $z^{\prime} / z$. falls when the precursor dications are unfolded before ECD by gentle infrared irradiation [Zubarev, R. A, unpublished results]. The IR-induced unfolding shortens the lifetime of the $\left[c^{\prime}+z \cdot\right]$ complex before its dissociation and, thus, lowers the probability of inter-chain $\mathrm{H}$. transfer. Similar unfolding can be obtained by increasing the electron energy or irradiation time (since even noncapture electron-ion interactions increase the ionic temperature). Therefore, relative $z^{\prime} / z$. measurements require fixed ECD conditions. All our data were collected at the following fixed conditions: the average electron energy in the center of the FT trap was $\approx 1.5 \mathrm{eV}$ and the irradiation time was $50 \mathrm{~ms}$. The work of $\mathrm{O}^{\prime}$ Connor et al., who observed H/D scrambling in two deuterated peptides is also a compelling evidence for path I and eq 2 [8]. An additional, theoretical argument against pathway II has been provided by Turecek, who calculated the radical $\mathrm{H}_{2} \mathrm{NCH}_{2} \mathrm{CONH}$. to be unstable [19].

Details of $z^{\prime}$ ion formation can be further probed by statistics of fragment size distributions. Changes in the $z^{\prime} / z$. ratio with the size of the neutral $c^{\prime}$ species and $z$. ions are shown in the main panel and inset of the left part of Figure 4, respectively.

The sensitivity of $\mathrm{H}$ - transfer rate to the size of $c^{\prime}$ neutrals (main panel) is much higher than to the size of the $z$. ions (inset). These results are rationalized as follows. The $z^{\prime} / z$. ratio depends upon the lifetime of the $\left[c^{\prime}+z \cdot\right]$ complex, which in turn depends upon the bonding strength inside this complex. The primary mechanism holding together $z$. ions and complementary $c^{\prime}$ neutrals is the solvation of the ionic charge on neutral groups of the $c^{\prime}$ species. An increased size of the neutral $c^{\prime}$ species offers more possibilities for efficient charge solvation of the $z$ ion. The bonding inside the $\left[c^{\prime}\right.$ $+z$ ] complex becomes stronger, and the lifetime of the complex increases. This explains the dramatic increase in the $z^{\prime} / z$. ratio in the main panel of Figure 4, left panel. On the other hand, as the size of the $z$. ion increases, more possibilities arise for intramolecular solvation within the $z$. ion itself, which effectively reduces the bonding strength inside the $\left[c^{\prime}+z \cdot\right]$ complex. This explains why the $z^{\prime} / z$. ratio drops with the size of the $z$. ion (Figure 4 , inset).

Since the identity of the amino acid C-terminal to the cleavage site influences the $z^{\prime} / z \cdot$ ratio, it is important to make sure that the size effect on the $z^{\prime} / z$. ratio is not a consequence of a particular amino acid distribution in tryptic peptides. Right panel of Figure 4 proves that the size effect is independent upon the amino acid identity. This panel presents the same statistics as the left panel, but separately for amino acids Gly, Asn, and Ala
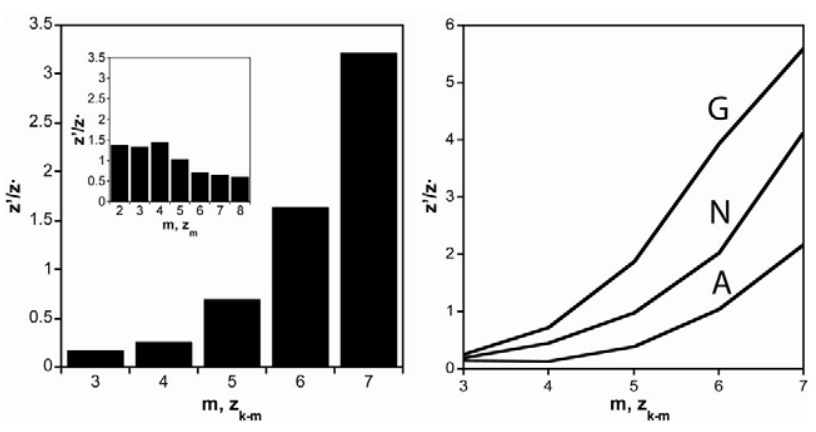

Figure 4. (Left) $z^{\prime} / z$. ratios as functions of the distance of the cleavage site $\mathrm{m}$ from the $\mathrm{N}$-terminus for $z_{k-m}$ ions, where $k$ is the peptide size, $m$ is also the size of the complementary $c^{\prime}$ neutral. Inset: the same for $z_{m}$ ions, where $m$ is now the size of the $z$-ion. (right), the same as in (left), but for three different amino acids C-terminal to the cleavage site, Gly, Asn, and Ala. 
C-terminal to the cleavage site (position $n$ ). Although the strength of the effect does depend upon the amino acid identity as would be expected from Figure 2, the trend is the same in all three cases, and is consistent with the general trend in the left panel. Thus, the size effect of the $c^{\prime}$ fragment acts independently of the identity of the amino acid in the $n$-th position.

In early ECD literature, it has been suggested that $\mathrm{H}$. transfer may be strongly affected by the proximity to the positive charge due to the high polarizability of the $\mathrm{H}$ atom [21]. This suggestion predicts rapidly growing $z_{m}{ }^{\prime} / z_{m}$. ratios for small $m$ as the distance between the cleavage site and the C-terminal charge decreases and generally higher sensitivity to the $z$. ion size compared with the size of $c^{\prime}$ neutrals. This prediction is in a disagreement with data in Figure 4, where reversed sensitivity order is observed and $z_{m}{ }^{\prime} / z_{m}$. ratio remains flat for $2 \leq m \leq 4$ (inset). Thus strong influence of the proximal charge on $\mathrm{H}$. transfer to $z$. ions finds no confirmation.

\section{H. Donor Groups}

The question, which chemical groups of $c^{\prime}$ neutrals donate hydrogen atom to $z$. ions, is important for understanding the overall mechanism of $\mathrm{H}$. transfer. Experimentally, it is best studied with deuterated samples [8], while the current study was based on data obtained in traditional proteomics experiments. Based on the variance analysis (Figure 2, inset), the prime candidate for the $\mathrm{H}$. donor is the amino acid adjacent to the cleavage site (position $n+1$ ). However, relatively modest sensitivity of $z^{\prime} / z$. values to the identity of the amino acid in that position and a somewhat elevated level of the variance for $(n-1)$ indicate that $\mathrm{H}$. rearrangement from $c^{\prime}$ to $z$. fragment may involve not only $(n+1)$ st residue, but also other residues of the $c^{\prime}$ fragment and perhaps even of the $z$. ion itself. In the latter case, intramolecular $\mathrm{H}$. transfer shifts the position of the radical to a more stable site. O'Connor et al. have explicitly assumed $\mathrm{H}$. transfer from residues remote of the cleavage site [8]. In another work, they argued that $\mathrm{H}$. can be easily abstracted from $\mathrm{C}_{\alpha}$ groups of amino acids, especially of glycines [18]. To test this hypothesis, $z^{\prime} / z$. ratios were calculated for $z_{k-m}$ ions containing glycine in different positions in the complementary $c$ fragment. To eliminate the size effect of $c_{m}{ }^{\prime}$, fixed value of its length, $m=5$, was chosen. To highlight the effect of $\mathrm{H}$. donation, the average value 0.79 of the $z^{\prime} / z \cdot$ ratio for $m=5$ was subtracted from all $z^{\prime} / z$ values. The results are presented in Figure 5.

While for Gly in the position $n+1$ the value in Figure 5 is positive (meaning higher than average propensity to donate hydrogen), for the same amino acid in other positions except the N-terminal position one obtains negative (below average) values. Similar calculations were performed for alanine, as well as glutamine and aspartic acid (Figure 5). Alanine shows lower than average propensity to donate hydrogen in

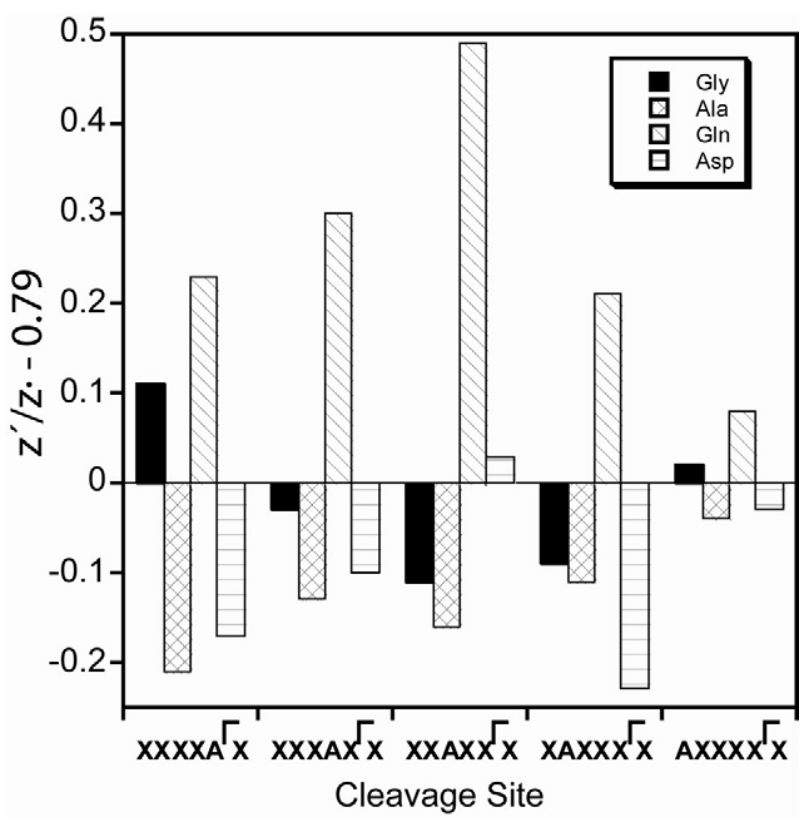

Figure 5. $z^{\prime} / z$. ratios for $z_{k-m}$ ions containing glycine, alanine, glutamine, and aspartic acid in different positions in the complementary $\mathrm{c}$ fragment. The length of $\mathrm{c}$ was fixed to five residues.

all positions. While aspartic acid behaves largely similar to alanine, glutamine is active in $\mathrm{H}$. donation in all positions. Thus, overall, glycine is not a particularly good donor of $\mathrm{H} \cdot$ atoms.

The above results highlight the fact that $\mathrm{H}$. donation depends not only on the nature of the amino acids but also on its distance to the cleavage site. This fact can be rationalized by the calculations by Rauk et al. of the energies of abstracting $\mathrm{H}$. from the $\alpha$-carbon atoms of amino acid residues in fully relaxed peptides as well as in $\alpha$-helices and $\beta$-sheets $[19,22,23]$. Using similar $a b$ initio computational technique as we (see below), these authors calculated that the $\mathrm{C}_{\alpha}-\mathrm{H}$ bond energy can be tuned by the local environment from about $345 \mathrm{~kJ} / \mathrm{mol}$ to $400 \mathrm{~kJ} / \mathrm{mol}$, i.e., in a broader range than the differences among various residues in fully relaxed peptides [23]. The modulation of the $\mathrm{C}_{\alpha}-\mathrm{H}$ bond strength can explain why the same amino acid in different positions has different effect on $\mathrm{H}$ - abstraction.

To quantify the overall impact on $\mathrm{H}$. transfer to $z$. ions of the presence of an amino acid AA in the $c$ fragment (as opposed to the presence of AA in the position $n+1$ in Figure 2) $z^{\prime} / z$. values of $z_{n-5}$ ions were calculated for two cases: when the five-residue long complementary $c^{\prime}$ fragment contained at least one amino acid AA, and when it contained none. The ratio of $z^{\prime} / z$. values in these two cases, $K(\mathrm{AA})$, is plotted in Figure 6 after subtracting unity to highlight higher-than-average and lower-thanaverage effects.

The strongest effect on $\mathrm{H}$ atom transfer was found for His and Gln, while the weakest one was for Asp and Ala. As expected from Figure 5, glycine again showed below-average overall donor propensity. This may seem as a disagreement with $\mathrm{H} / \mathrm{D}$ scrambling results of 


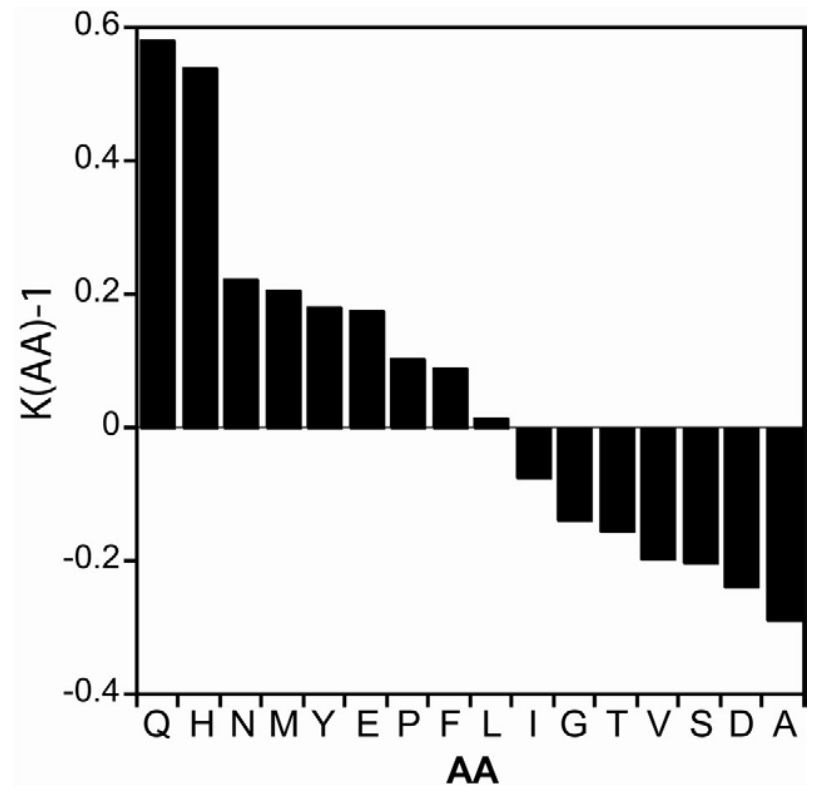

Figure 6. $\mathrm{z}^{\prime} / \mathrm{z}$ values of $z_{n-5}$ ions calculated for two cases: when the five-residue long complementary $\mathrm{c}$ fragment contained at least one amino acid AA, and when it contained none. The ratios of $z^{\prime} / z$. values in these two cases, $K(\mathrm{AA})$, are plotted after subtracting unity.

$\mathrm{O}^{\prime}$ Connor et al. [8]. But there are two $\mathrm{H}$ atoms on the $\alpha$-carbon atom of Gly as opposed to one for all other residues but proline (which has none), which doubles the effect of $\mathrm{H} / \mathrm{D}$ scrambling in glycine compared with other residues. On the other hand, the decreased H/D scrambling rate due to Asp N-terminal to the cleavage site noted by $\mathrm{O}^{\prime}$ Connor et al. [8] is in agreement with both Figures 2 and 6. Comparison of the $\mathrm{C}_{\alpha}-\mathrm{H}$ bond energies calculated by Rauk et al. [23] for amino acids in peptide chains (as opposed to free amino acids, see below) with data on Figure 6 showed only a modest anticorrelation ( $r=-0.43$, data not shown). The minus sign is habitually added by us to $r$ values to stress the negative character of anticorrelations [11]. The conclusion can be made from the results in Figure 6 that the side chains of residues with labile hydrogens may as much (or more) be the source of the transferred hydrogen atoms as the $\alpha$-carbon groups.

The amino acid in the $(n+1)$ st position may be a somewhat special case, since it is in close contact with the radical site on the $z$. ion. Not surprisingly, its effect on $\mathrm{H}$ - donation is the strongest (inset in Figure 2). Is the $\alpha$-carbon group of this amino acid in direct competition with the $\alpha$-carbon group of $n$-th amino acid for H.? If such competition were significant, it would lead to an anticorrelation between the $z^{\prime} / z$. ratios for positions $n$ and $(n+1)$ measured for the same amino acids, as the same residue that tends to gain $\mathrm{H} \cdot$ in $n$-th position would tend to retain it in the $(n+1)$ st position. In reality, a slight positive correlation exists $(r=+0.33)$ between the $z^{\prime} / z$. ratios for $n$ and $(n+1)$. For instance, Gly, and Ser promote $H \cdot$ transfer to $z \cdot$ in both $n$-th and $(n+1)$ st position, while His in either of these positions discourages such transfer. Thus, the $\alpha$-carbon radical of the $n$-th amino acid belonging to the $z$. ion competes for $\mathrm{H} \cdot$ not as much with the $\alpha$-carbon group of the $(n+1)$ st amino acid from the $c^{\prime}$ fragment as with other potential donor sites.

\section{Thermochemistry of H. Subtraction}

What determines different propensities to $\mathrm{H}$. transfer of different amino acids in $n$-th position? To reveal the degree to which the exothermicity of hydrogen abstraction by $z$. species is involved in determining this propensity, one needs to know $\mathrm{C}_{\alpha}-\mathrm{H}$ bond enthalpies for N-terminal amino acids in peptides or, in the absence of such data, at least for free amino acids. Rauk et al. have calculated the enthalpy values for four amino acids, Gly, Ala, Ser, and Thr [23]. Since this is not enough for quantitative comparison with our data and no additional information was available, we performed $a b$ initio calculations for twelve amino acid residues, including the above four, to increase the consistency of comparison. The energies were calculated as adiabatic energies of $\mathrm{H}$ - removal from the $\mathrm{C}_{\alpha}$ group, which are opposite in sign but equal in absolute values to the exothermicity of $\mathrm{H}$ - attachment to the $\alpha$-carbon radicals of $\mathrm{N}$-terminal amino acids in $z$. species. The source of $\mathrm{H}$. atoms was not explicitly assumed, but it were believed to be the same for all $z$. species. If thermochemistry were the driving force behind $\mathrm{H} \cdot$ abstraction, one would expect to have a correlation (although unlikely a perfect one) with between $\mathrm{H}$. abstraction propensities and enthalpy data. Such a correlation was indeed obtained (Figure 7a), and it was statistically valid, with the probability of obtaining such a value $(r=0.73)$ by pure chance below $1 \%$. Given that the accepted accuracy of the used calculations is $10 \mathrm{~kJ} / \mathrm{mol}[19,22,23]$, the obtained correlation is convincing enough evidence of the prevailing role of exothermicity of $\mathrm{H} \cdot$ attachment in $\mathrm{H} \cdot$ transfer rates.

The points of amino acids Glu, Gln, Asp, and Asn lie
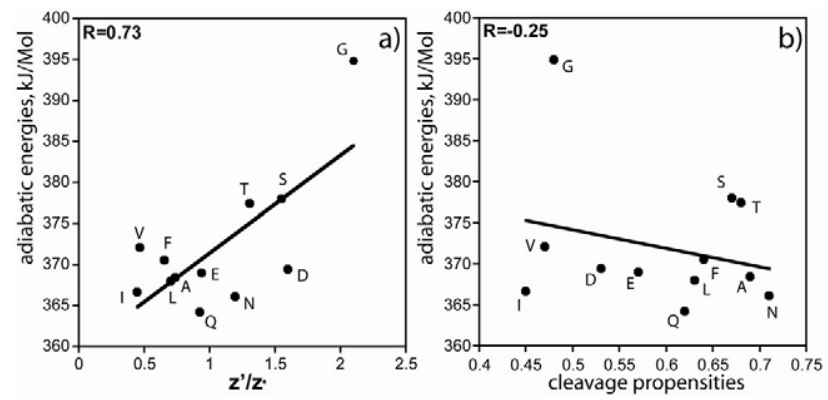

Figure 7. Adiabatic energies of formation of $\cdot$ A radicals from free amino acids by $\mathrm{H}$. abstraction from $\mathrm{C}_{\alpha}$-group calculated ab initio at B3LYP level of theory and using 6-31 + G(d) basis set as functions of (a) $z^{\prime} / z$. ratios taken from Figure 2 for residues in the position $n$, (b) propensities for $\mathrm{N}-\mathrm{C}_{\alpha}$ bond cleavage for $\mathrm{X}-\mathrm{A}$ bonds ( $\mathrm{X}$, any amino acid) taken from reference [11]. 
2 to $8 \mathrm{~kJ} / \mathrm{mol}$ below the general trend. The radicals of these amino acids tend to form in minimum-energy structures a hydrogen bond between the side chain and their own backbone amide, which stabilizes the radical by 5 to $10 \mathrm{~kJ} / \mathrm{mol}$. Formation of this H-bond in polypeptides may be hindered because of the involvement of the amide in intramolecular hydrogen bonding, and because it requires rotation of the side chain around the $\mathrm{C}_{\alpha}-\mathrm{C}$ bond by ca. $180^{\circ}$, which may be sterically impossible. Thus, the actual configuration of the radical that is primarily involved in $\mathrm{H}$ - transfer may, for these amino acids, not be the lowest-energy structure but a higherenergy conformer. If this is the case, the data points corresponding for these amino acids should come very close to the general trend and increase the correlation further. Indeed, exclusion of Glu, Gln, Asp, and Asn improves the correlation from 0.73 to 0.93 . At any rate, within most groups of similar amino acids, the tendency of increasing the $z^{\prime} / z$. ratio with the formation enthalpy is followed. This is true for pairs Ala/Gly, Ile/Leu, Gln/Asn, Glu/Asp, and Thr/Ser, and although for some of these pairs the difference in formation energies is small, the general character of the above tendency is clear. Thus, the main driving force for $\mathrm{H}$. abstraction by $z$. species from the complementary $c^{\prime}$ species is the process exothermicity, although entropic factors, such as steric effects and secondary structure also play an important role.

\section{Thermodynamics of $\mathrm{N}-\mathrm{C}_{\alpha}$ Bond Cleavage}

The calculated values of the energies of the formation of amino acid radicals provide a test whether thermodynamics plays as important a role in $\mathrm{N}-\mathrm{C}_{\alpha}$ bond cleavage as in $\mathrm{H}$. transfer. Ergodic dissociation kinetics is determined, besides entropy factors, by transition-state (TS) energies. In an important theoretical study, Turecek has found these to be rather low and very similar for various model systems representing peptide cations [19]..$^{\circ} \mathrm{He}^{\circ}$ has $^{\circ}$ noticed ${ }^{\circ}$ that $^{\circ}$ "the ${ }^{\circ} \mathrm{TS}^{\circ}$ energies ${ }^{\circ}$ for ${ }^{\circ} \mathrm{N}$ o- $\mathrm{C}_{\alpha}$ bond cleavage follow a trend ... that weaker bonds require lower ${ }^{\circ} S^{\circ}$ energies" "[19]. Thus, if thermodynamics is the main factor determining the rate of $\mathrm{N}-\mathrm{C}_{\alpha}$ bond dissociation, the bond strength is the most important parameter. Quoting the same work, "the ... N- $\mathrm{C}_{\alpha}$ bond dissociation energies depend on the nature of the leaving radical group. ... The stability of the radical product is the determining factor." Indeed, the strength $\mathrm{D}$ of an $\mathrm{N}-\mathrm{C}_{\alpha}$ bond in the sequence ... $\mathrm{X}_{n}+{ }_{2} \mathrm{X}_{n}+$ ${ }_{1}-A_{n} X_{n-1} \cdots(X$, any residue; $A$, specific residue $)$ is determined by the stability $U_{C}$ and $U_{Z}$. of the formed products $c^{\prime}$ and $z$. with $X_{n}+1$ and $A_{n}$ as terminal residues:

$$
\mathrm{D}=\mathrm{U}_{\mathrm{MH} \cdot}-\left(\mathrm{U}_{\mathrm{C}}+\mathrm{U}_{\mathrm{Z}}\right),
$$

where $U_{M H}$. is the stability of the peptide radical cation, precursor to $\mathrm{N}-\mathrm{C}_{\alpha}$ bond cleavage. The stability $\mathrm{U}_{\mathrm{C}}$ of the $c^{\prime}$ fragment can be considered constant, since con- tributions of individual amino acids $X_{i}$ in $c^{\prime}$ average in a large dataset to approximately the same value. Thus the bond strength $\mathrm{D}$ decreases with increased stability $U_{Z}$. of the fragment $\cdot A_{n} X_{n-1} \ldots$, which in turn depends primarily upon the stability of the radical $\cdot A_{n}$. Therefore, one would expect to find an anticorrelation between the cleavage frequency for $\mathrm{X}-\mathrm{A}$ bonds and the formation energy of the amino acid radicals $\cdot A$, just as the $\mathrm{H}$. transfer has manifested itself through the correlation ${ }^{\circ}$ with $^{\circ}$ radical $^{\circ}$ formation $^{\circ}$ energies $^{\circ}$ (Figure $\left.{ }^{\circ} 7 a\right) .{ }^{\circ}$ The bond cleavage frequencies for different amino acids in $n$-th ${ }^{\circ}$ position $^{\circ}$ were $^{\circ}$ obtained $^{\circ}$ from $^{\circ}$ reference $^{\circ}[11] . .^{\circ}$ An anticorrelation was indeed found with calculated bond strengths ${ }^{\circ}\left(r=-0.25,{ }^{\circ}\right.$ Figure $^{\circ} 7 \mathrm{~b},{ }^{\circ}$ or ${ }^{\circ} r=-0.20^{\circ}$ without Glu, Gln, Asp, and Asn), but it was too weak to be statistically significant. This result may be due to inaccuracies of the calculations. It may also be due to other major factors influencing the $\mathrm{N}-\mathrm{C}_{\alpha}$ cleavage rate besides the TS energies. If, for instance, formation of radical sites in the precursor ions is not random as was implicitly assumed, but amino acid specific, this specificity would destroy the correlation.

\section{$H \cdot$ Loss}

The loss of hydrogen from $z$. ions is an altogether different process from the $\mathrm{H} \cdot$ attachment. If these processes were related, then the residue in the position $n$ promoting $\mathrm{H}$. loss from $z$. would discourage its attachment to $z$, and vice versa. In reality, no valid anticorrelation is found between these propensities. Amino acids Ser, Thr, and Trp in position $n$ promote both $\mathrm{H}$. attachment to and the $\mathrm{H}$. loss from the $z$. fragment (Figures ${ }^{\circ}{ }^{\circ}$ and 3 )..${ }^{\circ} t^{\circ}$ is $^{\circ}$ thus ${ }^{\circ}$ unlikely ${ }^{\circ}$ that ${ }^{\circ} \mathrm{H} \cdot{ }^{\circ} \operatorname{loss}^{\circ}$ from ${ }^{\circ} z$. involves $\mathrm{H}$. transfer to $c^{\prime}$ neutrals, which are evenelectron species and thus have relatively low $\mathrm{H}$ - affinity. It is more likely that $\mathrm{H}$. loss occurs as additional mechanism of energy relaxation in $z \cdot$ ions after the $\left[c^{\prime}+\right.$ $z \cdot]$ complex undergoes separation. Disproportional abundant losses of two $\mathrm{H} \cdot$ atoms from Ser, Thr, and Trp as well as the complete absence of triple losses indicates that the double-H. loss may occur via a concerted mechanism involving $\mathrm{H}_{2}$ formation. The high exothermicity of $\mathrm{H}_{2}$ formation may be the driving force behind the double $\mathrm{H}$. loss.

\section{Conclusions}

Occurrence of hydrogen rearrangement to/from $z \cdot$ ions, a well known phenomenon in ECD, has been quantified for the first time using a large dataset. The high overall frequency of occurrence found (47\%) is not particularly surprising, given what was known on hydrogen rearrangement ${ }^{\circ}{ }^{\circ}$ radical $^{\circ}$ cations $^{\circ}$ of $^{\circ}$ other biomolecules ${ }^{\circ}$ [24]. These findings lend support to ideas related to radical cascades $[16]^{\circ}$ as $^{\circ}$ secondary ${ }^{\circ}$ processes ${ }^{\circ}$ occurring ${ }^{\circ}$ after ${ }^{\circ}$ the primary $\mathrm{N}-\mathrm{C}_{\alpha}$ bond cleavage that yields "classical" $c^{\prime}$ and $z$. species. The driving factor for $\mathrm{H} \cdot$ abstraction by $z$. ions from the complementary $c^{\prime}$ species is the process 
exothermicity, but on a more detailed level the rate is affected by structural features of the fragmenting ions. Thus, $z^{\prime} / z$. ratios carry information on the secondary and possibly high level structure of gas-phase polypeptides. Exploring this information requires more knowledge than is currently available. Ab initio calculations favoring nonergodic mechanism of $\mathrm{N}-\mathrm{C}_{\alpha}$ bond cleavage are definitely not a final word on the issue, but they may be a useful addition to the discussion. Detailed information on the occurrence frequency of hydrogen attachment and loss will be important for understanding peptide radical chemistry, a rapidly growing research area. In practice, detailed knowledge of secondary reactions in backbone fragments will facilitate automatic interpretation of ECD mass spectra in protein identification and characterization, including de novo sequencing.

\section{Acknowledgments}

This work was supported by the Knut and Alice Wallenberg Foundation and Wallenberg Consortium North (grant WCN2003UU/SLU-009 and instrumental grant for FTMS) as well as the Swedish research council (grants 621-2004-4897, 621-2002-5025, and 621-2003-4877). Thomas Köcher and Christopher Adams are acknowledged for insightful discussion.

\section{References}

1. Link, A. J.; Eng, J.; Schieltz, D. M.; Carmack, E.; Mize, G. J.; Morris, D. R.; Garvik, B. M.; Yates, J. R. Direct Analysis of Protein Complexes Using Mass Spectrometry. Nat. Biotechnol. 1999, 17, 676-682.

2. Pandey, A.; Mann, M. Proteomics to Study Genes and Genomes. Nature 2000, 405, 837-846.

3. Aebersold, R.; Mann, M. Mass Spectrometry-Based Proteomics. Nature 2003, 422, 198-207.

4. Zubarev, R. A.; Kelleher, N. L.; McLafferty, F. W. Electron Capture Dissociation of Multiply Charged Protein Cations. A Nonergodic Process. J. Am. Chem. Soc. 1998, 120, 3265-3266.

5. Nielsen, M. L.; Savitski, M. M.; Zubarev, R. A. Improving Protein Identification Using Complementary Fragmentation Techniques in Fourier Transform Mass Spectrometry. Mol. Cell. Proteomics 2005, 4, 835845.

6. Savitski, M. M.; Nielsen, M. L.; Kjeldsen, F.; Zubarev, R. A. ProteomicsGrade de Novo Sequencing Approach. J. Proteome Res. 2005, 4, 2348 2354.

7. Huang, Y. Y.; Triscari, J. M.; Tseng, G. C.; Pasa-Tolic, L.; Lipton, M. S. Smith, R. D.; Wysocki, V. H. Statistical Characterization of the Charge
State and Residue Dependence of Low-Energy CID Peptide Dissociation Patterns. Anal. Chem. 2005, 77, 5800-5813.

8. O'Connor, P. B.; Lin, C.; Cournoyer, J. J.; Pittman, J. L.; Belyayev, M.; Budnik, B. A. Long-Lived Electron Capture Dissociation Product Ions Experience Radical Migration via Hydrogen Abstraction. J. Am. Soc. Mass Spectrom. 2006, 17, 576-585.

9. Adams, C. M.; Kjeldsen, F.; Zubarev, R. A.; Budnik, B. A.; Haselmann, K. F. Electron Capture Dissociation Distinguishes a Single D-Amino Acid in a Protein and Probes the Tertiary Structure. J. Am. Soc. Mass Spectrom. 2004, 15, 1087-1098.

10. Kjeldsen, F.; Savitski, M. M.; Adams, C. M.; Zubarev, R. A. Localization of Positive Charges in Gas-Phase Polypeptide Polycations by Tandem Mass Spectrometry. Int. J. Mass Spectrom., 2006, 252, 204-212.

11. Savitski, M. M.; Kjeldsen, F.; Nielsen, M. L.; Zubarev, R. A. Complementary Sequence Preferences of Electron Capture Dissociation and Vibrational Excitation in Fragmentation of Polypeptide Polycations. Angew. Chem. Int. Ed., 2006, 45, 5301-5303.

12. Zhang, R. Q.; Huang, J. H.; Bu, Y. X.; Han, K.; Lee, S. T.; He, G. Z. An Effective Scheme for Selecting Basis Sets for Ab Initio Calculations. Chin. Acad. J. 2000, 43, 375-388.

13. Dolgounitcheva, O.; Zakrzewski, V. G.; Ortiz, J. V. Structures and Electron Detachment Energies of Uracil Anions. Chem. Phys. Lett. 1999, 307, 220-226.

14. Cooper, H. J.; Hudgins, R. R.; Hakansson, K.; Marshall, A. G. Secondary Fragmentation of Linear Peptides in Electron Capture Dissociation. Int. J. Mass Spectrom. 2003, 228, 723-728.

15. Mirgorodskaya, E.; Roepstorff, P.; Zubarev, R. A. Localization of O-Glycosylation Sites in Peptides by Electron Capture Dissociation in a Fourier Transform Mass Spectrometer. Anal. Chem. 1999, 71, 4431-4436.

16. Zubarev, R. A.; Horn, D. M.; Fridriksson, E. K.; Kelleher, N. L.; Kruger, N. A.; Lewis, M. A.; Carpenter, B. K.; McLafferty, F. W. Electron Capture Dissociation for Structural Characterization of Multiply Charged Protein Cations. Anal. Chem. 2000, 72, 563-573.

17. Horn, D. M.; Zubarev, R. A.; McLafferty, F. W. Automated de Novo Sequencing of Proteins by Tandem High-Resolution Mass Spectrometry. Proc. Natl. Acad. Sci. U.S.A. 2000, 97, 10313-10317.

18. Leymarie, N.; Costello, C. E.; O'Connor, P. B. Electron Capture Dissociation Initiates a Free Radical Reaction Cascade. J. Am. Chem. Soc. 2003, $125,8949-8958$.

19. Turecek, F. N-C- $\alpha$ Bond Dissociation Energies and Kinetics in Amide and Peptide Radicals. Is the Dissociation a Nonergodic Process? J. Am. Chem. Soc. 2003, 125, 5954-5963.

20. Turecek, F.; Syrstad, E. A. Mechanism and Energetics of Intramolecular Hydrogen Transfer in Amide and Peptide Radicals and Cation Radicals. J. Am. Chem. Soc. 2003, 125, 3353-3369.

21. Zubarev, R. A.; Kruger, N. A.; Fridriksson, E. K.; Lewis, M. A.; Horn, D. M.; Carpenter, B. K.; McLafferty, F. W. Electron Capture Dissociation of Gaseous Multiply-Charged Proteins is Favored at Disulfide Bonds and Other Sites of High Hydrogen Atom Affinity. J. Am. Chem. Soc. 1999, 121, 2857-2862.

22. Rauk, A.; Yu, D.; Armstrong, D. A. Toward Site Specificity of Oxidative Damage in Proteins: $\mathrm{C}-\mathrm{H}$ and $\mathrm{C}-\mathrm{C}$ Bond Dissociation Energies and Reduction Potentials of the Radicals of Alanine, Serine, and Threonine Residues-An ab Initio Study. J. Am. Chem. Soc. 1997, 119, 208-217.

23. Rauk, A.; Yu, D.; Taylor, J.; Shustov, G. V.; Block, D. A.; Armstrong, D. A. Effects of structure on $\mathrm{C}-\alpha-\mathrm{H}$ Bond Enthalpies of Amino Acid Residues: Relevance to H Transfers in Enzyme Mechanisms and in Protein Oxidation. Biochemistry 1999, 38, 9089-9096.

24. McLafferty, F. W.; Turecek, F. Interpretation of Mass Spectra. Sausalito, CA: University Science Books, 1993. 